\title{
Cost Analysis of Current Grids and its Implications for Future Grid Markets
}

\author{
Marcel Risch, Jörn Altmann \\ International University in Germany, School of Information Technology, \\ Campus 3, 76646 Bruchsal, Germany \\ \{Marcel.Risch, Jorn.Altmann\}@i-u.de
}

\begin{abstract}
Commercial Grid markets have been a topic of research for many years. Many claims about the advantages of trading computing resources on markets have been made. However, due to a lack of Grid computing offerings, these claims could not be verified. This paper analyzes the question whether using the Grid is financially advantageous, using the Amazon.com EC2 service as a reference. To perform this analysis, the costs of computing resources in different usage scenarios are calculated, if Grid resources and in-house resources are used. The comparison of the costs reveals that while the Grid is cheaper in the short term, it is not a good investment in the long term and, thus, the existence of a Grid economy will not lead to an end of ownership but rather to a reduction in in-house resources and more efficient resource usage.
\end{abstract}

Keywords: Commercial Grids, Grid Computing, Business Models, Cost Modeling, Capacity Planning, Grid Economics, Utility Computing, Markets.

\section{Introduction}

Commercial Grids have been a focal point of research for many years. The idea of selling idle computing resources on a market for computational power has been advocated since the early 1960s. With the advent of the Internet and the Internet economy, this idea once again received attention during the last years. The advantages of Grid markets have been emphasized with various claims that could not be validated, as a Grid economy did not exist. However, such an economy has now started to develop with the introduction of a number of cluster or cloud computing providers, who sell compute resources on a pay-per-use basis. Using these offers as a basis, we are now able to validate some of the frequently made claims about commercial Grids.

In this paper, we will focus on four claims. They address the financial advantages that companies would gain from using a commercial Grid. Since companies are, in general, seeking ways to gain competitive advantage or to lower their operational costs, the financial advantages of Grids play a major role in promoting Grid usage. Therefore, we will analyze the following four claims:

- Claim 1: Companies can reduce the staff for maintaining resources. This idea has been propagated in research and commercial circles [1][2][3]. 
- Claim 2: Companies have large computational power available at their fingertips on a pay-per-use basis [4][5].

- Claim 3: Companies do not have to purchase the resources and, thus, have no cost of ownership [2][3][4], which is significant for high-performance computing resources.

- Claim 4: The advantages of commercial Grids are the reduction in cost [4][5].

Although Claim 1 is difficult to validate for all enterprises, we believe that, due to the difficulty of using Grid resources, it is highly unlikely that an increased Grid usage will result in major savings from personnel reductions in medium-sized companies. There are a number of issues that indicate that the headcount in the inhouse IT support staff will remain unchanged. Firstly, the software running on a Grid resource must be maintained and monitored in the same way as the software running on in-house resources. Secondly, the in-house staff must be able to handle many different virtualization tools, such as Xen [6], VMWare [7]. For each of these tools used in a Grid market, the in-house staff must maintain and create the correct images. Since there are as of now no support tools available, the in-house staff must be knowledgeable in may different virtualization tools. Thirdly, any company using the Grid has to perform a detailed cost-benefit analysis to determine whether using the Grid is more cost effective than purchasing in-house resources. Since this analysis requires intimate knowledge of all applications, hardware, and the skill to predict the load levels, experienced staff is needed.

Due to these reasons, we do not believe that the size of in-house IT staff for medium-sized companies can be reduced due to increased Grid usage. Small enterprises, on the other hand, will not only require the computational power, they will also need some software to run on these computers. For these companies, the Grid is more interesting if it offers Software-as-a-Service and not just pure computing power. For large companies, the cost savings through Grid usage are very little. Large companies already benefit from the economies of scale in the operation of their IT resources. IT resources of large enterprises are organized in a few data centers, supported by a sufficiently large number of in-house IT staff. Therefore, any outsourcing of the data center service (i.e. using the Grid) could not result in significant cost savings. Since no type of enterprise is expected to reduce its IT support staff headcount through the use of Grid computing, we will not include the personnel costs in the following parts of this analysis.

Continuing with Claim 2, we can state that this claim is obviously true: All currently existing Grid resource providers have a pricing structure, in which the customer only pays for the computational power used. Furthermore, while there are some limits imposed on the number of resources available, in general, these limits are fairly broad and should not pose any difficulties for users.

Claim 3 is also trivial to verify. Since Grid resources are not purchased but are rather rented to the buyer, the buyer has no costs of ownership. Since Grid resources are not owned by the purchasing company, the purchasing company does not have any costs of ownership.

To perform the analysis of Claim 4, we need to consider the market structure, the type of resources sold and the size of the enterprise using the Grid. Based on this information and evidence gathered in the existing commercial Grid environment, we 
will determine during the remainder of this paper whether this claim can be supported.

This paper is structured as follows: In section 2, we analyze the potential Grid users, the types of resources available, and the current market structure. In the third section, we present some basic cost information for Grid and in-house resources. In addition, we characterize three companies that are used for comparing Grid and in-house resource costs. These companies need to acquire additional resources, which have the characteristics of one basic instance of an Amazon EC2 resource [8]. In section 4, we present a case-by-case cost analysis for different Grid usage scenarios. In the fifth section, we will analyze the results of the case study and draw some conclusions about the structure of the future Grid market. Finally, we conclude by presenting some open items, which can be explored in future research.

\section{Analysis Framework}

\subsection{Potential Grid Users}

For our analysis, we assume that commercial enterprises mainly use the Grid. These enterprises can be categorized as follows: i) home offices; ii) small enterprises; iii) medium-sized enterprises; and iv) large enterprises. The definitions are standardized in the European Union [9]. In addition to this, we also define the companies in terms of their IT expertise. In general, the smaller the company, the less IT expertise it has. In other words, home offices and small enterprises have less IT expertise than medium-sized or large enterprises. Therefore, their needs for IT solutions differ. Thus, home offices and small enterprises need more complete solutions (e.g. Software-as-a-Service) for their IT needs than medium-sized or large enterprises. Large enterprises, which can perform any kind of IT investment and already benefit from the economies of scale (which Grid computing promises), would not get any additional benefit from participating in a commercial Grid.

Therefore, for our analysis, we only consider medium-sized enterprises. Those companies are characterized by restricted budget for IT investments, and the existence of an IT department.

\subsection{Resource Types Available on a Grid}

In general, any type of compute resource can be sold on a Grid market. However, for the purpose of the analysis, a classification of those resources helps highlighting the characteristics of those resources. Our classification of computing resources resulted in the following four groups:

- High Performance Computers (HPC): Supercomputers for specialized tasks and compute-intensive applications.

- Server clusters: A number of servers, which are located in the same facility and interconnected to ensure high communication speeds between the individual 
servers. They can be used for high-performance computing as well as for monolithic applications as the computing resources of the next group.

- Servers: Individual servers for running monolithic applications.

- Desktops: Individual workstations for employees.

In this paper, we will focus solely on individual servers, since the existing computing cloud offerings (e.g. Amazon EC2 service) is aimed at companies requiring additional servers. This also makes the comparison between in-house resources and Grid resources easier, since prices for in-house resources can be easily obtained from various hardware manufacturers.

\subsection{The Market Structure}

The structure of the current Grid market is an oligopoly. We have only a few large providers in the market, such as Amazon.com EC2 [8], Sun Grid [10], and Tsunamic Technologies [11]. Because of this market structure and slight differentiation of their services, they can set their prices such that it maximizes profits.

An alternative market structure would be characterized by complete competition between resource providers, who sell their excess resources on a cost basis. In such a market, prices would be generally lower due to competition and the only price fluctuations would be caused by high demand. The demand for resources is higher than the available resources on the Grid.

In this paper, we will focus on the current market and more specifically, on resources obtained from Amazon.com's EC2 service. This provider was chosen for a number of reasons. Firstly, it started its resource sales shortly after the advent of the GridEcon Project [12]. Secondly, the pricing structure is very well described, making it easy to calculate the prices for different usage scenarios. Thirdly, the provider was chosen for its clear specification of the virtual machines, thus ensuring that equivalent servers for in-house installation can be found easily. Lastly, the Amazon EC2 service was chosen due to its popularity: According to [13], this service is now used by about 60,000 customers and generates a revenue of about $\$ 131$ million.

\section{Methodology and Data Collection}

To determine in which cases the Grid is cheaper than in-house resources, we will use three companies, called $C_{1}, C_{2}$ and $C_{3}$, which require additional resources in the form of a single server. Furthermore, we will assume a linear growth of costs for all companies, i.e. if the price for a single server is $\mathrm{P}$, then the cost for $\mathrm{n}$ servers will be $\mathrm{n} * \mathrm{P}$. Economies of scale are neglected, since it is difficult to estimate both the point at which they set in and the magnitude of the discount.

The three companies will obtain their resources as follows: Company $C_{1}$ will purchase its server for in-house installation expensively. Company $C_{1}$ is assumed to be a small company with little purchasing power. Company $\mathrm{C}_{1}$ has higher costs than company $C_{2}$ which also obtains its resources for in-house installation. Company $C_{2}$ is 
assumed to be bigger and, therefore, has higher purchasing power. Company $\mathrm{C}_{3}$ will purchase resources on the Amazon EC2 service.

Since company $\mathrm{C}_{3}$ uses the Amazon EC2 resources, the resources used by the other companies should be comparable. In particular, we will assume that all servers have at least a $2 \mathrm{GHz}$, single-core $\mathrm{CPU}$, at least $2 \mathrm{~GB}$ of main memory and a hard disk with at least 200GB storage. To match the requirements, company C3 will purchase another 40GB of storage from Amazon's S3 service [14].

The prices for the resources used by companies $\mathrm{C}_{1}$ and $\mathrm{C}_{2}$ were obtained using the online tools of Dell [15], Gateway [16], and HP [17]. Based on the prices found, company $C_{1}$ has been assigned a server, which is $25 \%$ more expensive than the most expensive model. This price was chosen to ensure that $C_{1}$ has the highest costs and thus, has the largest incentive for using the Grid. Company $\mathrm{C}_{2}$, on the other hand, pays the average price for all resources, thus ensuring that the price paid by $C_{2}$ is realistically chosen.

The prices that company C3 faces have been obtained from the Amazon EC2 Web site. The actual prices for all resources are described in chapter 4 . Since the Amazon.com cost structure emphasizes usage times, we will assume that a server is used continuously for 30 days. This will be the basis for the comparison in chapter 5 .

Since the costs of Amazon.com's EC2 depend on the actual usage of the resources, we have to introduce usage scenarios which take the actual usage into account. We have decided on the four scenarios listed below. These were chosen because they illustrate different generic usage patterns that may be encountered by SMEs using the Grid. These scenarios also illustrate the effect the pricing structure has on the overall Grid cost. The term "upload bandwidth" refers to the data transferred out of the Amazon.com EC2 service and the term "download bandwidth" refers to the data transferred into the service.

- Scenario 1: Update Server: The server uses a lot of upload bandwidth and little download bandwidth. Such a server would be used for companies with many customers

- Scenario 2: Backup Server: The resource uses a lot of download bandwidth and less upload bandwidth. This type of server would be used for off-site backups for important data.

- Scenario 3: Computational Server: The resource uses little bandwidth as it is mainly used for computations.

- Scenario 4: Medium-Sized Enterprise Web Server: A server that is barely used but hosts a vital program for the company, such as a Web server.

There is one additional alternative to using commercial Grids: Virtual Private Server (VPS) hosting. There are a number of providers of this type of service; however, the resources offered are geared more towards web hosting rather than computation. This is made obvious by the lack of resource specification when it comes to processor speeds. Instead, customers are attracted by the amount of storage offered and the main memory size.

We have compared a number of VPS providers, such as EMC [18], InMotion [19] and Yourserving.com [20]. We have found that the resources most comparable to the ones offered by the Amazon.com EC2 service cost between \$90 and \$170 per month, depending on the subscription length and the provider. Since these costs are significantly above the costs for in-house resources and since the bandwidth 
allowances are sometimes severely restricted, we have decided that this type of service is not an adequate replacement for in-house resources or for Amazon.com EC2 resources. Therefore, we have ignored this service type in our analysis.

\section{Cost Calculation}

Under normal circumstances, resources will be written off after three years using normal depreciation rules. This means that every month, a depreciation cost is incurred which is added to the other monthly costs. In our cost calculation, we will simplify matters by assuming that depreciation is not used, but rather that the entire cost of an in-house resource has to be paid upfront. This allows the reader to see when the Grid usage costs reach or exceed the costs for in-house resources.

In this section, we introduce the costs that the three companies face. In addition, we will calculate the monthly costs for each of the two companies that use in-house resources.

\subsection{Company $\mathrm{C}_{1}$}

As we have stated earlier, company $C_{1}$ obtains its resources expensively. The following is a list of costs that $\mathrm{C}_{1}$ will have to pay for its resources.

- New server: From our research, we have found that an expensive new server costs no more than $\$ 650$. We will assume that $C_{1}$ will have to pay $\$ 800$, which is more than $25 \%$ more than the highest price we found.

- Electricity: According to the Energy Information Administration (EIA) [21], the electricity cost for commercial enterprises is at most $14.65 \mathrm{ct} / \mathrm{KWh}$ in the contiguous US. For $\mathrm{C}_{1}$, we will assume an electricity cost of $20.00 \mathrm{ct} / \mathrm{KWh}$. To calculate the monthly electricity usage costs, we need to determine the power consumption for the server. Power supplies usually range between 200W and $500 \mathrm{~W}$; since the resource used in our calculation is a server which does not require power-hungry components, we will assume that the power supply is in the middle of this range. Therefore, we chose a $350 \mathrm{~W}$ power supply which means that the server uses $350 \mathrm{~W} / \mathrm{h}$. This means that one hour of operating the server costs $0.350 K W H * 0.20 \frac{\$}{K W H}=0.07 \$$.

In the first month, the company will have to pay both the server and the electricity. The costs are shown in Table 1. For the following months, only the electricity costs must be paid which means that the monthly costs are at $\$ 50.40$.

Table 1. First month costs for $\mathrm{C}_{1}$

\begin{tabular}{|l|r|r|r|}
\hline & Quantity & \multicolumn{1}{|c|}{ Price } & Total (\$) \\
\hline Hardware Purchase & 1 & $800 \$$ & 800.00 \\
\hline Electricity & $720 \mathrm{~h}$ & $0.07 \$ / \mathrm{h}$ & 50.40 \\
\hline \multicolumn{3}{|l|}{} & 850.40 \\
\hline Total (first month) & &
\end{tabular}




\subsection{Company $\mathrm{C}_{2}$}

A similar calculation has to be performed for company $\mathrm{C}_{2}$. This company is able to obtain its resources and electricity cheaper than company $\mathrm{C}_{1}$, thus having a competitive advantage without the Grid. The costs of $\mathrm{C}_{2}$ are divided as follows:

- New server: The average server price for the Amazon EC2-type server was about $\$ 500$. We will assume that company $\mathrm{C}_{2}$ paid this price for its resources.

- Electricity: Using the EIA table again, we decided to use a more realistic electricity price. Since a large number of IT companies is located in the California, we decided to the use the average commercial electricity price for 2007 as a reference. At time of writing of this paper, the price was $12.76 \mathrm{ct} / \mathrm{KWh}$, which was rounded to $13 \mathrm{ct} / \mathrm{KWh}$ for easier computation. Using an online power calculator [22], we determined that a server would use about 200W. This means that one hour of operating the server costs $0.200 K W H * 0.13 \frac{\$}{K W H}=0.026 \$$.

Using this information, we can now calculate the usage costs for the first month for company $\mathrm{C}_{2}$. This information is given in Table 2 .

Table 2. First month costs for $\mathrm{C}_{2}$

\begin{tabular}{|c|c|c|c|}
\hline & Quantity & Price & Total (\$) \\
\hline Hardware Purchase & 1 & $500 \$$ & 500.00 \\
\hline Electricity & $720 \mathrm{~h}$ & $0.026 \$ / \mathrm{h}$ & 18.72 \\
\hline Total (first month) & & & 518.72 \\
\hline
\end{tabular}

We can see that company $C_{2}$ has much lower costs than company $C_{1}$. In addition, it should be noted that the monthly costs are less than half of the costs incurred by $\mathrm{C}_{1}$, namely only $\$ 18.72$.

\subsection{Company $\mathrm{C}_{3}$}

Finally, the costs for company $\mathrm{C}_{3}$ need to be introduced. Since $\mathrm{C}_{3}$ uses the Amazon services EC2 [8] and S3 [14], the total cost incurred for each month depends on the actual usage. In Table 3, the costs for the various items are shown.

Table 3. Costs for using Amazon.com EC2 and S3

\begin{tabular}{|l|l|l|}
\hline Item & Cost & Restrictions \\
\hline Hourly cost & $0.10 \$ / \mathrm{CPU}-\mathrm{hr}$ & None \\
\hline Data Transfer In & $0.10 \$ / \mathrm{GB}$ & None \\
\hline Data Transfer Out & $0.18 \$ / \mathrm{GB}$ & First $10 \mathrm{~TB} /$ month \\
\hline Data Transfer Out & $0.16 \$ / \mathrm{GB}$ & Next $40 \mathrm{~TB} /$ month \\
\hline Data Transfer Out & $0.13 \$ / \mathrm{GB}$ & Over 50TB/month \\
\hline Hard Disk Space & $0.15 \$ / \mathrm{GB}-$ month & Each GB over $160 \mathrm{~GB}$ \\
\hline
\end{tabular}




\section{Cost Comparison of Each Scenario}

In this section, we determine the costs incurred by using the Grid in each of the four scenarios and compare it with the cost of in-house purchases. These scenarios are defined in the form of usage characteristics.

\subsection{Scenario 1: Download Server}

The download server uses a large amount of upload bandwidth and very little download bandwidth. As a basis for our calculation, we used some data from the SecondLife Blog [23]. We assumed that the download server would be used heavily for four days and then be used less for the remainder of the month. Since the blog referred to 70GB of downloads per hour for almost one day which was then followed by several days of 30GB per day, we decided on the following upload quantities: 70GB for the first day, 30GB for the following 3 days and 3.5GB for the remaining 26 days:

$$
70 \frac{G B}{h r} * 24 h r+30 \frac{G B}{h r} * 24 h r s * 3+3.5 \frac{G B}{h r} * 624 h r=6024 G B
$$

The result was rounded to 6000GB for the 30 day period to simplify the calculation. The entire cost for the first 30 days of operating a Grid resource is calculated in Table 4.

Table 4. Download server costs

\begin{tabular}{|c|c|c|c|}
\hline & Quantity & Price & Total (\$) \\
\hline CPU-hrs & 720 & $0.10 \$ / h$ & 72 \\
\hline Hard Disk Space & 40 & $0.15 \$ / G B$ & 6 \\
\hline Upload Data & 6000 & $0.18 \$ / G B$ & 1080 \\
\hline Download Data & 100 & $0.10 \$ / G B$ & 10 \\
\hline Total & & & 1168 \\
\hline
\end{tabular}

It can be easily seen that the Grid in this case is extremely expensive, mainly due to the high upload costs. Comparing this value with the prices obtained by companies $C_{1}$ and $C_{2}$, we can see that $C_{3}$ pays $125 \%$ more than the amount paid by $C_{2}$ and $37 \%$ more than $\mathrm{C}_{2}$.

However, in this scenario, we assume that $C_{1}$ and $C_{2}$ have sufficient bandwidth to satisfy the download requirements. Since this amount of bandwidth is usually not available for medium-sized companies, both $\mathrm{C}_{1}$ and $\mathrm{C}_{2}$ would have to purchase additional bandwidth. In order to support 70GB/day in uploads; they would need about 14 lines of an AT\&T 6Mbit download line service [24]. This costs $\$ 60$ per line and therefore, the total cost for 14 lines would be $\$ 840$ per month. Alternatively, a Verizon 15Mbit upload line could be purchased for about \$240 per month [25].

In both cases, the monthly cost for companies $C_{1}$ and $C_{2}$ would increase. However, in the long-term the Grid would still be more expensive. This can be demonstrated by showing the cost graphs of all companies. In Figure 1, we show the cost graphs over time if both $C_{1}$ and $C_{2}$ use the more expensive Internet access service. If the 
companies would use the less expensive option the slope of the curve would be even lower.

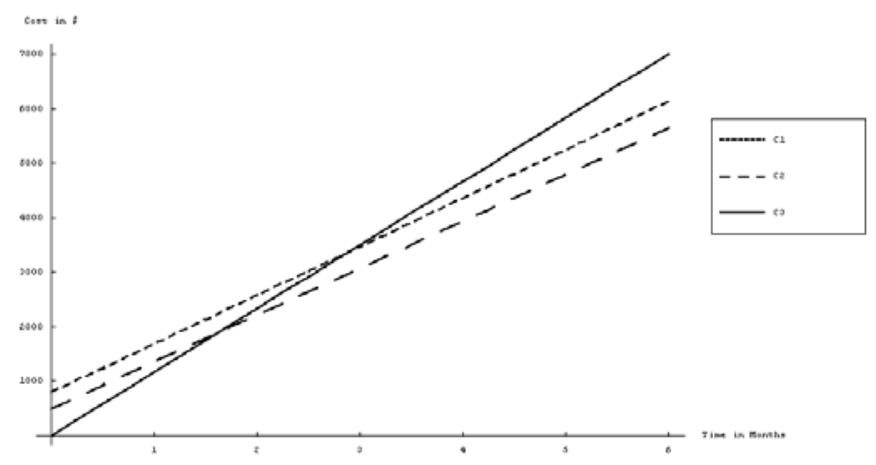

Figure 1. Price comparison with expensive internet

As we can see, even with the expensive Internet access, the costs of the Grid are higher than for in-house resources after three months. It should be noted however, that the Internet prices require a one-year subscription. If the company requires the high download bandwidth for one month only, then the Grid would be much cheaper, since the Internet connection would cost at least \$2900 for a one-year subscription.

\subsection{Scenario 2: Backup Server}

A backup server has a high number of downloads and a low number of uploads, assuming that the data stored in the backup server is rarely needed. Since the upload bandwidth is not used as much as in the first case, the companies using in-house resources would not have to resort to purchasing additional Internet connectivity. For company $\mathrm{C}_{3}$, we will assume that it performs uploads of 500GB every month. This corresponds to loosing two complete sets of data and making some minor corrections. Furthermore, we assume that the company downloads about 3000GB. This corresponds to backing up 100GB every day and replacing copies after two days. The monthly cost calculation can be seen in Table 5 .

Table 5. Monthly costs of a backup server

\begin{tabular}{|c|c|c|c|}
\hline & Quantity & Price & Total (\$) \\
\hline CPU-hrs & 720 & $0.10 \$ / \mathrm{h}$ & 72 \\
\hline Hard Disk Space & 40 & $0.15 \$ / G B$ & 6 \\
\hline Upload Data & 500 & $0.18 \$ / G B$ & 90 \\
\hline Download Data & 3000 & $0.10 \$ / G$ & 300 \\
\hline Total & & & 468 \\
\hline
\end{tabular}

In this case, the Grid is cheaper than in-house resources in the beginning as well. However, as the monthly costs are much higher when using the Grid, company $\mathrm{C}_{3}$ soon pays more than the companies purchasing in-house resources. After about 1.5 
months, company $\mathrm{C}_{2}$ will pay less than company $\mathrm{C}_{3}$; after 2.5 months company $\mathrm{C}_{1}$ will pay less than company $\mathrm{C}_{3}$. This development is shown in Figure 2.

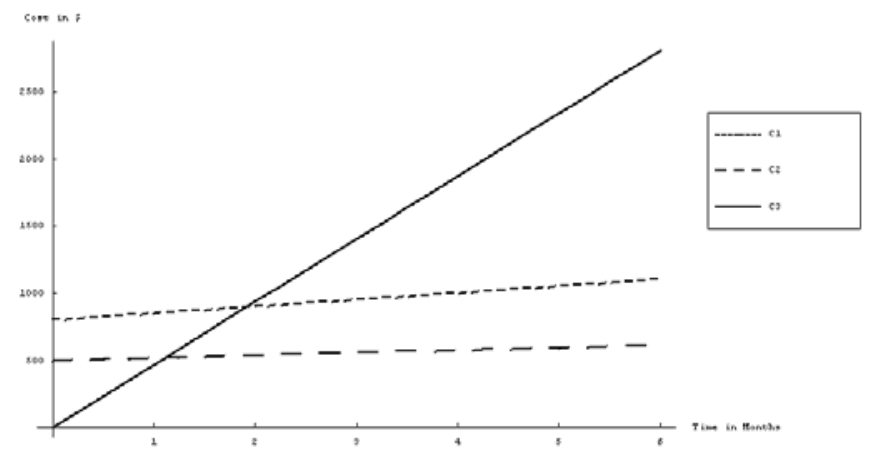

Figure 2. Price comparison for backup servers

The figure illustrates how much more expensive the Grid is in the long run. However, it should be noted that this calculation is only valid if the company who uses in-house resources can host the new server. If the new server has to be hosted at a different location, which is not owned by the company, or if such a location has to be built or bought, then the Grid will be cheaper, since the costs for the new location will be much higher than the monthly Grid costs.

\subsection{Scenario 3: Computational Server}

So far, we have only examined resources that require large amounts of bandwidth. However, bandwidth is one of the main cost drivers of the Amazon EC2 service. Therefore, we will now focus on a server which requires less bandwidth and is largely used for compute-intensive tasks. We assume that the server requires 100GB upload and 100GB download, since this server may be part of a computationally large workflow where the individual subjobs transfer data between each other. The monthly Grid costs are detailed in Table 6.

Table 6. Computational Server monthly costs

\begin{tabular}{|c|c|c|c|}
\hline & Quantity & Price (\$) & Total (\$) \\
\hline CPU-hrs & 720 & $0.10 \$ / h$ & 72 \\
\hline Hard Disk Space & 40 & $0.15 \$ / G B$ & 6 \\
\hline Upload Data & $100 \mathrm{~GB}$ & $0.18 \$ / G B$ & 18 \\
\hline Download Data & $100 \mathrm{~GB}$ & $0.10 \$ / G B$ & 10 \\
\hline Total & & & 106 \\
\hline
\end{tabular}

Although the expenses for Internet access are still significant, the monthly cost is much lower than that of the previous scenario. Even when using less bandwidth, the Grid is still more expensive than in-house resources in the medium-term. The cost difference between the bandwidth-intensive servers and this server is reflected by the 
fact that the breakeven point between Grid resources and the in-house resources has been moved to a later date: for company $\mathrm{C}_{2}$, the breakeven is reached after slightly less than six months, for company $\mathrm{C}_{1}$ the breakeven is reached after a little more than 14 months. This is illustrated in Figure 3 below.

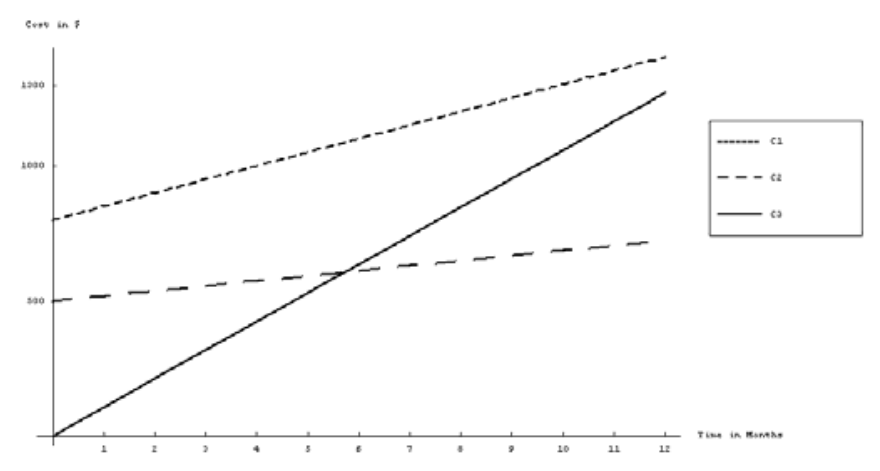

Figure 3. Price comparison for computational servers

\subsection{Scenario 4: SME Web Server}

In this case, we assume that a small, little-known company uses the Grid to set up a Web server. Since the company is not known, there will be very little traffic on the server, and therefore, these will be almost no bandwidth used. However, since Amazon.com charges the user for each started GB of bandwidth used, we will take some minimal traffic into account. During the first month, we will assume some higher download usage, since the machine image will have to be transferred. For the subsequent months, we will assume that only web traffic will be incurred. For this traffic, we will assume that each web page has a size of about $100 \mathrm{~KB}$ and that the ten pages are request per day. This means that about 30MB of data transferred out of Amazon.com.

Furthermore, we will assume that the company will not purchase additional hard disk space on Amazon's S3 service. Only the costs for the subsequent months are shown in Table 7, the costs for the first month are only slightly and can therefore be neglected.

Table 7. SME web server first month costs

\begin{tabular}{|l|r|r|r|}
\hline & Quantity & Price & \multicolumn{1}{c|}{ Total (\$) } \\
\hline CPU-hrs & 720 & $0.10 \$ / \mathrm{h}$ & 72.00 \\
\hline Upload Data & 1 & $0.18 \$ / \mathrm{GB}$ & 0.18 \\
\hline Download Data & 1 & $0.10 \$ / \mathrm{GB}$ & 0.10 \\
\hline Total & \multicolumn{3}{|r}{} \\
\hline
\end{tabular}


These costs are significantly lower than those for of the previous scenarios. Thus, the Grid price in this case is much more competitive than in the previous cases. This fact is illustrated in Figure 4 below.

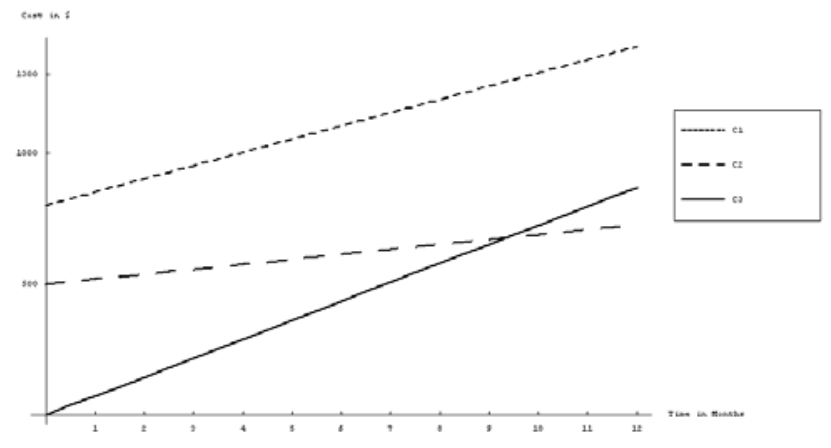

Figure 4. Cost comparison for a SME web server

Compared to the resources bought by company $\mathrm{C}_{2}$, the Grid becomes more expensive after about 9 months. Since company $C_{1}$ has higher costs, the breakeven will not be reached until about month 35. Therefore, we can conclude that the Grid is cheaper in the medium- to long-term if the in-house resources are very expensive. However, if the in-house resources are cheap, then the Grid is only cheaper in the medium-term.

\section{Analysis}

This section will consist of two parts: in the first part, we will discuss the remaining claim using the information provided in section five. In the second part, we will determine the implications that the analysis results have on the future Grid market.

\subsection{Claim Analysis}

In the introduction to this paper, we have given four claims about Grid economies. Of these we have already addressed three claims, namely the claim that companies have large computational power available at a pay-per-use model, the claim that companies can reduce their in-house staff, and the claim of no cost of ownership.

In the last claim, it was asserted that the Grid reduces the cost for hardware resources. This claim has been the starting point for the detailed cost analysis in the previous section. From our calculations, we can state that using the Grid is not always cheaper than using in-house resources. In fact, every company must determine for itself at which point the Grid becomes too expensive. In general, the costeffectiveness of the Grid depends on two parameters: the usage duration and the usage intensity. Using these parameters, we can enumerate some cases, in which the Grid usage would be advantageous: 
- To cover short, infrequent demand peaks. These peaks should not occur more often than once every several months, or once every year. The peaks last for a few weeks at most.

- If the data backup should be made in a physically different location, which cannot be afforded by a company otherwise.

- Lightly used resources over a short to medium-term period.

Since there are cases, in which the currently existing Grid market is not cheaper than in-house resources, we can conclude that the existence of a Grid economy will not lead to an end of ownership. But, companies will be able to reduce their resource infrastructure by covering infrequent usage peaks with Grid resources. However, regularly occurring peaks must still be provided for using in-house resources. Consequently, there will still be in-house resources that remain idle for some periods of time. Two general statements can be made, based on our calculations:

- For heavily used resources: If the resource requirements exceed the in-house capacity for less than two months during a depreciation period of three years, then the current Grid market is cheaper. Therefore, given the current resource prices, the usage duration is the main decision factor for which resources should be bought. If the resource is used for less than $6 \%$ of the three years (depreciation period), it should be bought on the Grid.

- For less heavily used resources: If the resource requirements exceed the in-house capacity for less than six months during the three year depreciation period, then the current Grid market is cheaper. Therefore, if the resource is used for less than $17 \%$ within a period of three years, it should be bought on the Grid.

\subsection{Implications for the Future Grid}

We have determined that companies will still have excess of in-house resources. Therefore, a solution needs to be found as to what can be done with these resources when they are idle. There are two courses of action open to companies: They either turn off the resources to conserve electricity and thereby reduce expenditures, or, they sell the excess resources on a Grid market for commodity goods. The first option is sub-optimal, since only the electricity costs are reduced. Since electricity at this point remains cheap, the savings will be fairly low. The second course of action, on the other hand, will allow companies to recoup most of their costs, including maintenance and depreciation costs. This added income would allow the company to leave its resources switched on while at the same time ensuring that no money is lost due to idle resources.

A Grid market for commodity goods, in which companies can sell their idle resources, would be characterized by intense competition between resource sellers. The advantages of such a market are numerous: Due to the intense competition, the prices would be lower than in the current Grid market, which is a seller's market. This lower price would, in turn, encourage buyers to purchase more Grid resources, since the difference in cost between the Grid resources and their in-house resources is relatively small. It would also lower the barrier of entry to the Grid for new users. In addition, Green IT objectives are met, since resources are used to their full capacity and, therefore, resources rarely sit idle any longer. 
A Grid market as described above has to fulfill some requirements: Firstly, it has to sell commodity goods, which are comparable and substitutable. Therefore, the market allows for a competitive market environment. Secondly, the Grid market has to be able to manage many providers and buyers in a single platform. It must be able to handle a large volume of trades and store large amounts of data about these trades. Due to the competition, resource providers will use marginal pricing for their resources to remain competitive. Only congestion which is caused by short term high demand peaks will cause high prices.

For such a market to operate smoothly, some support services need to be developed. These services are especially important for companies that have little or no Grid expertise. This idea has been at the heart of the GridEcon project which has developed a framework to support services for SMEs with little Grid expertise. These services include various brokers (e.g. Risk Broker, Workflow Broker, and Insurance Broker) as well as services such as a Capacity Planning Service. The goal of these services is to simplify the transition to the Grid and its usage as much as possible.

\section{Conclusion}

In this paper, we have discussed four claims about Grid computing, and analyzed one in detail. We have found that the Grid is not always cheaper than in-house resources. Since, at present, only few Grid resource providers exist in the market, they can easily generate profits. Therefore, the effects of the economies of scale are negated. Therefore, any company considering the use of Grid resources should carefully calculate whether the Grid is actually cheaper than in-house resources.

From the analysis of the costs of the current Grid (which is a set of data centers of servers), we have determined that the existence of a Grid will not lead to an end of ownership but will lead to a decrease in over-provisioning of computing resources. We expect that rare demand peaks will be covered using Grid resources.

Since, under the current market structure, companies still have to over-provision, they will have to face the question of what to do with idle resources. Selling these resources on a Grid market is the best option, since all incurred costs can be recouped. If many companies sell their idle resources on a market, this will lead to strong competition, which will force prices to remain low unless there is a severe resource shortage. The low prices will attract more buyers, thereby increasing supply and demand. During times of high demand, companies may even be able to make small profits due to the increased prices they can charge.

The workings of this competitive market need to be studied further, with special attention paid to price setting, the price development over time, the actions taken by resource sellers and buyers, and the effects these actions have on the market. This also leads to the question of how companies will act and react to price fluctuations.

In addition, the analysis performed in this paper can also be repeated for other resource types, such as differentiated goods. The results could form the basis for a Grid markets for differentiated goods. 


\section{References}

[1] Southern Partnership for Advanced Computational Infrastructures (SPACI), http://www.spaci.it/content.php?loc=projects\&pg=prj.php\&cat=gm\&id=10

[2] Yeo, C.S., Buyya, R., Assunção, M.D., Yu, J., Sulistio, A., Venugopal, S., Placek, M.: Utility Computing on Global Grids. In: Bidgoli, H. (eds.) The Handbook of Computer Networks, chapter 143. John Wiley \& Sons, New York, USA (2007).

[3] Fujitsu Siemens Computers, http://www.fujitsusiemens.it/it_trends/grid_computing.html, 2008.

[4] Altmann, J., Courboubetis, C., Darlington, J., Cohen, J.: GridEcon - The Economic-Enhanced Next-Generation Internet. In: GECON 2007, LNCS vol. 4685. Springer, Heidelberg (2007).

[5] Altmann, J., Ion, M., Mohammed, A. A. B.: Taxonomy of Grid business models. GECON 2007, LNCS 4685, Springer, Heidelberg, 2007.

[6] XenSource, Inc., http://xen.org/, 2008.

[7] VMware, Inc, http://www.vmware.com/, 2008.

[8] Amazon Elastic Compute Cloud (Amazon EC2), http://www.amazon.com/gp/browse.html?node=201590011, 2008.

[9] Enterprise - $\quad$ SME http://ec.europa.eu/enterprise/enterprise_policy/sme_definition/index_en.ht m, 2008.

[10] Sun Grid, http://www.sun.com/service/sungrid/index.jsp, 2008.

[11]Tsunamic Technologies Inc., http://www.clusterondemand.com/, 2008.

[12] GridEcon, http://www.gridecon.eu, 2008.

[13] Schonfeld, E.: Who Are The Biggest Users of Amazon Web Services? It's Not Startups. http://www.techcrunch.com/2008/04/21/who-are-the-biggestusers-of-amazon-web-services-its-not-startups/, 2008.

[14]Amazon Simple Storage Service (Amazon S3), http://www.amazon.com/gp/browse.html?node=16427261, 2008.

[15]Dell, http://www.dell.com/, 2008.

[16] Gateway, Inc., http://www.gateway.com/, 2008.

[17]Hewlett-Packard Development Company, L.P. , http://www.hp.com/, 2008.

[18]EMC Telecom Corporation, http://www.emcwebhosting.com/vps_hosting_extreme_linux.php, 2008.

[19]InMotion Hosting, Inc , http://www.inmotionhosting.com/vps_hosting.html, 2008.

[20]yourserving.com , http://yourserving.com/vps_server, 2008.

[21]Energy Information Administration, Average Retail Price of Electricity to Ultimate Customers by End-Use Sector, by State, http://www.eia.doe.gov/cneaf/electricity/epm/table5_6_b.html, 2008.

[22]Journey Systems, LLC., Power Calculator, http://www.journeysystems.com/?powercalc, 2008.

[23]Linden Research, Inc, $\quad$ SecondLife Blog, http://blog.secondlife.com/2006/10/26/amazon-s3-for-the-win/, 2008. 
[24]AT\&T,

https://swot.sbc.com/swot/dslMassMarketCatalog.do?do=dslProductPage\& offerId=90027\&serviceType=DYNAMICIP, 2008.

[25]Verizon,

http://www22.verizon.com/content/businessfios/packagesandprices/package sandprices.htm, 2008. 